\title{
氨基取代苯并咪唑衍生物的合成及在酮的氢转移反应中的应用
}

\author{
段凯李小娜李云庆王家喜* \\ (河北工业大学化工学院 天津 300130)
}

\begin{abstract}
摘要 以 $\alpha$-氨基酸与邻苯二胺在微波辐射下反应合成了 $\alpha$-氨基取代苯并咪唑(1 - 5). 1-(1H-苯并 $[\alpha]$ 咪唑-2-基)乙胺(1)与 溴丁烷反应可形成单丁基、二丁基、三丁基取代产物 1a $\sim 1 d, 1$ 的氨基经 Boc 保护, $N$-烷基化后制备咪唑环上的 $N$-烷基 化产物 $1 \mathrm{i} \sim 1 \mathrm{~g}$. 制备的氨基取代咪唑与 $\mathrm{Ru}(\mathrm{II})$ 化合物原位组成催化体系, 考察了其在取代苯乙酮的氢转移反应中的催 化活性. 结果表明 $\mathrm{RuCl}_{2}\left(\mathrm{PPh}_{3}\right)_{3}$ 与各配体组成的催化剂均有较好的催化活性, 含有 $\mathrm{NH}_{2}$ 基团的 $\alpha$-氨基取代苯并咪㟇化 合物参与的催化体系催化活性最好, TOF (Turnover frequency)可达到 $40200 \mathrm{~h}^{-1}$.
\end{abstract}

关键词 氨基酸; 苯并咪唑; $N$-烷基化; 微波; 钓络合物; 酮; 氢转移反应

\section{Syntheses and Application of Aminated Benzimidazole Derivatives in Transfer Hydrogenation Reaction of Ketones}

\author{
Duan, Kai Li, Xiaona Li, Yunqing Wang, Jiaxi* \\ (School of Chemical Engineering, Hebei University of Technology, Tianjin 300130)
}

\begin{abstract}
A series of $\alpha$-amino-benzimidazole derivatives $\mathbf{1} \sim \mathbf{5}$ were synthesized by reaction of $\alpha$-amino acid with $o$-phenylenediamine under microwave irradiation. 1-(1H-Benzo[d]imidazol-2-yl)ethanamine (1) reacted with bromobutane forming mono-butyl, dibutyl and tributyl substituted benzimidazole $\mathbf{1 a} \sim \mathbf{1 d}$; and the $N$-alkylated derivatives $\mathbf{1 i} \sim \mathbf{1 g}$ were synthesized by protection of amine group by Boc and then $N$-alkylation. The catalytic capability of the catalyst generated in situ from $\mathrm{Ru}(\mathrm{II})$ compound and obtained benzimidazole derivatives were evaluated in the transfer hydrogenation of ketones using iso-PrOH as hydrogen donor and solvent. The results revealed that catalyst generated in situ from $\mathrm{RuCl}_{2}\left(\mathrm{PPh}_{3}\right)_{3}$ and ligands had moderate to high activity. The catalyst system with $\alpha$-amino-benzimidazole derivative containing $\mathrm{NH}_{2}$ group is the most active and the highest TOF (turnover frequency) was up to $40200 \mathrm{~h}^{-1}$.
\end{abstract}

Keywords amino acid; benzimidazole; $N$-alkylation; microwave; $\mathrm{Ru}(\mathrm{II})$ complex; ketone; transfer hydrogenation

不饱和化合物如羰基化合物及亚胺的还原反应在 醇、胺的制备反应中非常重要, 氢转移反应是以氢给予 体代替氢气进行还原的反应 ${ }^{[1]}$. 氢转移反应所用的催化 剂中以 $\mathrm{Ru}^{[2]}, \mathrm{Rh}^{[3]}$ 及 $\mathrm{Ir}^{[4]}$ 络合物最多, 其中含 $\mathrm{N}-\mathrm{H}$ 官能 团的络合物表现出良好的催化活性. 近年来 Baratta 及 Ohkuma 小组 ${ }^{[5]}$ 发现含氨基吡啶的钓络合物在不对称氢 转移反应中具有很高的反应活性, Sandoval 小组发现钓 与氨基咪唑衍生物及有机膦的混合物在苯乙酮的不对 称催化氢化反应中有高的不对称选择性, 这种高不对称 选择性可能是手性膦与手性胺的协同作用诱导的 ${ }^{[6]}$. 苯 并咪唑氨基酸衍生物与钉组成的催化剂对苯乙酮的不 对称氢转移反应具有较好的催化性能 ${ }^{[7]}$, 而仅用氨基取
代咪唑为配体的氢转移反应未见报道. 本文利用邻苯二 胺与 $\alpha$-氨基酸在微波作用下的无溶剂反应合成了相应 的氨基取代苯并咪唑，经 $N$ 烷基化反应合成出系列氨基 取代的咪唑衍生物, 考察了所得咪唑衍生物的结构对其 在钓催化的苯乙酮氢转移反应中的催化性能.

\section{1 实验部分}

\section{1 试剂与仪器}

试剂: 化学试剂均为分析纯直接使用. $\mathrm{RuCl}_{2}\left(\mathrm{PPh}_{3}\right)_{3}$ 的制备参考文献[8], m.p. $132 \sim 134{ }^{\circ} \mathrm{C},{ }^{31} \mathrm{P}$ NMR (162 $\left.\mathrm{MHz}, \mathrm{CDCl}_{3}\right) \delta: 29.8$ (s).

仪器: 气相色谱仪(山东鲁南瑞虹化工仪器有限公

\footnotetext{
*E-mail: jwang252004@126.com

Received March 30, 2012; revised May 7, 2012; published online May 11, 2012

Project supported by the Natural Science Foundation of Hebei Province of China (No. B2011202087)

河北省自然科学基金(No. B2011202087)资助项目.
} 
司 SP-6890, 毛细管柱, 固定相: CP-Chirasil-Dex CB; 数 据处理: N2000 色谱数据工作站, 浙江大学智达信息工 程研究所); 核磁共振谱仪 (瑞士布鲁克公司 AVANCE400); 红外光谱仪(布鲁克公司 TENSOR27); 显微熔点测定仪(北京科仪电光仪器厂 XT4-100A); 微 波实验仪(南京汇研微波系统工程有限公司 MG08S-2B), 元素分析仪(德国 Elementar 公司 Vario EL)

\section{$1.2 \alpha$-氨基取代苯并咪唑衍生物的合成}

\subsubsection{1-(1H-苯并 $[d]$ 咪唑-2-基)乙胺 $(1)$ 的合成}

$100 \mathrm{~mL}$ 中单口瓶中加入磷酸 $5 \mathrm{~mL}$ 、多聚磷酸 $2 \mathrm{~mL}$ 、 丙氨酸 $2.67 \mathrm{~g}(0.03 \mathrm{~mol}) 、$ 邻苯二胺 $3.24 \mathrm{~g}(0.03 \mathrm{~mol})$, 室 温下搅拌均匀. 放入微波反应器中, $80 \mathrm{~mA}(255 \mathrm{~W})$ 下间 歇反应, TLC 检测反应进程. 反应完毕后加入 $30 \mathrm{~mL}$ 水 稀释, 用 $\mathrm{Ca}(\mathrm{OH})_{2}$ 调 $\mathrm{pH}$ 至 $8 \sim 9$, 过滤. 滤液减压浓缩, 所得固体通过硅胶柱层析(淋洗剂-乙酸乙酯), 得到 4.12 $\mathrm{g}$ 淡黄色固体, 收率 $85 \%$. m.p. $169 \sim 171{ }^{\circ} \mathrm{C}$ (文献值 ${ }^{[9]}$ : $\left.168 \sim 170{ }^{\circ} \mathrm{C}\right) ;{ }^{1} \mathrm{H}$ NMR $\left(400 \mathrm{MHz}, \mathrm{CDCl}_{3}\right) \delta: 7.58 \sim 7.56$ $(\mathrm{m}, 2 \mathrm{H}), 7.24 \sim 7.21(\mathrm{~m}, 2 \mathrm{H}), 4.47(\mathrm{q}, J=6.8 \mathrm{~Hz}, 1 \mathrm{H})$, 1.60 (d, $J=7.2 \mathrm{~Hz}, 3 \mathrm{H})$; IR (KBr) v: 3366, 3051, 2975, 2891, 2734, 1932, 1882, 1775, 1620, 1590, 1530, 1456, 1420, 1311, 1273, 1221, 1124, 1062, 1020, 991, 933, 850, $750 \mathrm{~cm}^{-1}$. Anal. calcd for $\mathrm{C}_{9} \mathrm{H}_{11} \mathrm{~N}_{3}: \mathrm{C} 67.06, \mathrm{H} 6.88, \mathrm{~N}$ 26.07; found C 67.15, H 6.74, N 26.12.

\subsubsection{1-(1H-苯并 $[d]$ 咪唑-2-基)-2-甲基丙胺 $(2)$ 的合} 成

方法同第 1.2 .1 节. 缴氨酸 $3.51 \mathrm{~g}(0.03 \mathrm{~mol})$ 与邻苯 二胺 $3.24 \mathrm{~g}(0.03 \mathrm{~mol})$ 微波下反应, 制得 $4.70 \mathrm{~g}$ 淡黄色 固体, 收率 83\%. m.p. 183 $184{ }^{\circ} \mathrm{C} ;{ }^{1} \mathrm{H}$ NMR $(400 \mathrm{MHz}$, $\left.\mathrm{CDCl}_{3}\right) \delta: 7.59 \sim 7.57(\mathrm{~m}, 2 \mathrm{H}), 7.24 \sim 7.22(\mathrm{~m}, 2 \mathrm{H}), 4.16$ $(\mathrm{d}, J=5.0 \mathrm{~Hz}, 1 \mathrm{H}), 2.38 \sim 2.32(\mathrm{~m}, 1 \mathrm{H}), 0.99(\mathrm{~d}, J=6.9$ $\mathrm{Hz}, 3 \mathrm{H}), 0.93$ (d, $J=6.8 \mathrm{~Hz}, 3 \mathrm{H})$; IR (KBr) v: 3423, 3374, 3081, 3052, 2958, 2870, 2739, 1936, 1894, 1620, 1590, $1538,1455,1432,1307,1272,1230,1027,935,749,732$ $\mathrm{cm}^{-1}$. Anal. calcd for $\mathrm{C}_{11} \mathrm{H}_{15} \mathrm{~N}_{3}$ : C 69.81, H 7.99, N 22.20; found C 69.60, H 7.90, N 22.50.

1.2.3 1-(1H-苯并 $[d]$ 咪唑-2-基)-2-苯基乙胺 $(3)$ 的合 成

方法第同第 1.2 .1 节. 苯丙氨酸 $4.95 \mathrm{~g}(0.03 \mathrm{~mol})$ 与 邻苯二胺 $3.24 \mathrm{~g}(0.03 \mathrm{~mol})$ 微波下反应, 后用 $\mathrm{Ca}(\mathrm{OH})_{2}$ 调 $\mathrm{pH}$ 至 $8 \sim 9$, 有大量固体生成, 过滤. 将滤饼用乙醇洗两 次, 乙醇相减压浓缩, 所得固体用乙酸乙酯重结晶, 得 到 $5.03 \mathrm{~g}$ 淡黄色固体, 收率 71\%. m.p. 178 $180{ }^{\circ} \mathrm{C} ;{ }^{1} \mathrm{H}$ NMR $\left(400 \mathrm{MHz}, \mathrm{CDCl}_{3}\right) \delta: 7.60 \sim 7.58(\mathrm{~m}, 2 \mathrm{H}), 7.35 \sim$ $7.31(\mathrm{~m}, 2 \mathrm{H}), 7.28 \sim 7.23(\mathrm{~m}, 5 \mathrm{H}), 4.53(\mathrm{dd}, J=9.5,4.1$ $\mathrm{Hz}, 1 \mathrm{H}), 3.52$ (dd, $J=13.7,4.1 \mathrm{~Hz}, 1 \mathrm{H}), 2.92$ (dd, $J=13.7$,
$9.5 \mathrm{~Hz}, 1 \mathrm{H})$; IR (KBr) v: 3386, 3302, 3081, 3026, 2915, 2852, 2753, 2673, 1931, 1890, 1812, 1769, 1619, 1590, $1538,1454,1425,1358,1315,1275,1227,1099,1029$, $931,751,699 \mathrm{~cm}^{-1}$. Anal. calcd for $\mathrm{C}_{15} \mathrm{H}_{15} \mathrm{~N}_{3}: \mathrm{C} 75.92, \mathrm{H}$ 6.37, N 17.71; found C 76.18, H 6.27, N 17.47.

\subsubsection{1,3-(1H-苯并 $[d]$ 咪唑-2-基)-1-丙胺(4)的合成}

方法同第 1.2 .1 节. 谷氨酸 $4.41 \mathrm{~g}(0.03 \mathrm{~mol})$ 与邻苯 二胺 $3.24 \mathrm{~g}(0.03 \mathrm{~mol})$ 微波下反应后, 制得 $6.92 \mathrm{~g}$ 淡黄 色固体, 收率 $79 \%$. m.p. $124 \sim 126{ }^{\circ} \mathrm{C}$ (文献值 ${ }^{[10]}$ : 123 $\left.125{ }^{\circ} \mathrm{C}\right) ;{ }^{1} \mathrm{H}$ NMR (400 MHz, DMSO- $\left.d_{6}\right) \delta: 7.49 \sim 7.47$ $(\mathrm{m}, 4 \mathrm{H}), 7.14 \sim 7.09(\mathrm{~m}, 4 \mathrm{H}), 4.12(\mathrm{q}, J=6.8 \mathrm{~Hz}, 1 \mathrm{H})$, $2.94 \sim 2.90(\mathrm{~m}, 2 \mathrm{H}), 2.39 \sim 2.34(\mathrm{~m}, 1 \mathrm{H}), 2.23 \sim 2.14(\mathrm{~m}$, 1H); IR (KBr) v: 3359, 3174, 3096, 3061, 2930, 2862, 2780, 1930, 1625, 1537, 1442, 1322, 1275, 1220, 1112, 1026, $737 \mathrm{~cm}^{-1}$. Anal. calcd for $\mathrm{C}_{17} \mathrm{H}_{17} \mathrm{~N}_{5}$ : C 70.08, $\mathrm{H}$ 5.88, N 24.04; found C 70.03, H 5.90, N 24.12.

\section{2 .5 2-(吡咯烷-2-基)-1H-苯并 $[d]$ 咪唑(5)的合成}

方法同第 1.2 .1 节. 脯氨酸 $3.45 \mathrm{~g}(0.03 \mathrm{~mol})$ 与邻苯 二胺 $3.24 \mathrm{~g}(0.03 \mathrm{~mol})$ 微波下反应，制得到 $4.65 \mathrm{~g}$ 淡黄色 固体 ${ }^{[11]}$, 收率 $83 \%$. m.p. 166 $167{ }^{\circ} \mathrm{C} ;{ }^{1} \mathrm{H}$ NMR (400 $\left.\mathrm{MHz}, \mathrm{CDCl}_{3}\right) \delta: 7.57 \sim 7.55(\mathrm{~m}, 2 \mathrm{H}), 7.24 \sim 7.21(\mathrm{~m}, 2 \mathrm{H})$, $4.60(\mathrm{dd}, J=8.4,5.7 \mathrm{~Hz}, 1 \mathrm{H}), 3.15 \sim 3.02(\mathrm{~m}, 2 \mathrm{H}), 2.38 \sim$ $2.31(\mathrm{~m}, 1 \mathrm{H}), 2.18 \sim 2.10(\mathrm{~m}, 1 \mathrm{H}), 1.88 \sim 1.81(\mathrm{~m}, 2 \mathrm{H})$; IR (KBr) v: 3417, 3151, 3051, 2973, 2873, 2680, 1932, 1892, $1771,1620,1590,1538,1454,1428,1324,1310,1272$, $1223,1092,1028,935,749,741 \mathrm{~cm}^{-1}$. Anal. calcd for $\mathrm{C}_{11} \mathrm{H}_{13} \mathrm{~N}_{3}$ : C 70.56, H 7.00, N 22.44; found $\mathrm{C} 70.63, \mathrm{H}$ $6.94, \mathrm{~N} 22.42$.

\subsection{1-(1H-苯并 $[d]$ 咪唑-2-基)乙胺(1)与溴丁烷反应合 成 $1 \mathrm{a} \sim 1 \mathrm{~d}$}

$100 \mathrm{~mL}$ 单口瓶中加入 $11.61 \mathrm{~g}(0.01 \mathrm{~mol}), \mathrm{KOH} 1.20$ $\mathrm{g}(0.02 \mathrm{~mol}), 50 \mathrm{~mL}$ 异丙醇, 常温搅拌 $0.5 \mathrm{~h}$ 后加入溴丁 烷 $2.06 \mathrm{~g}(0.015 \mathrm{~mol})$, 在 $85{ }^{\circ} \mathrm{C}$ 下回流反应, TLC 检测反 应进程. $48 \mathrm{~h}$ 后, 停止反应, 过滤, 滤液减压浓缩后经硅 胶柱层析 [淋洗剂: $V$ (乙酸乙酯 $): V($ 石油醚 $)=1: 4$ 后加 大极性至 $V($ 乙酸乙酯 $): V($ 石油醚 $)=1: 2$ ]分离, 制得产 物 $\mathbf{1 a} \sim \mathbf{1 d}$.

$N$-[1-(1H-苯并 $[d]$ 咪坐-2-基)乙基]-1-丁胺(1a)：粘稠 液体 $0.43 \mathrm{~g}$, 收率 $20 \% .{ }^{1} \mathrm{H}$ NMR $\left(400 \mathrm{MHz}, \mathrm{CDCl}_{3}\right) \delta$ : $7.72 \sim 7.71(\mathrm{~m}, 2 \mathrm{H}), 7.37 \sim 7.34(\mathrm{~m}, 2 \mathrm{H}), 5.12$ (q, $J=6.8$ $\mathrm{Hz}, 1 \mathrm{H}), 3.21 \sim 2.88(\mathrm{~m}, 2 \mathrm{H}), 2.10(\mathrm{~d}, J=6.9 \mathrm{~Hz}, 3 \mathrm{H})$, $2.05 \sim 1.78(\mathrm{~m}, 2 \mathrm{H}), 1.42 \sim 1.29(\mathrm{~m}, 2 \mathrm{H}), 0.92 \sim 0.78(\mathrm{~m}$, $3 \mathrm{H}) ;{ }^{13} \mathrm{C} \mathrm{NMR}\left(101 \mathrm{MHz}, \mathrm{CDCl}_{3}\right) \delta: 148.2,123.8,53.0$, 45.6, 28.3, 19.9, 17.7, 13.4; IR (KBr) v: 3424, 3153, 2964, $2785,1534,1455,1270,752 \mathrm{~cm}^{-1}$. Anal. calcd for 
$\mathrm{C}_{13} \mathrm{H}_{19} \mathrm{~N}_{3}$ : C 71.85, H 8.81, N 19.34; found C 72.01, H 8.78, N 19.20 .

$N$-[1-(1-丁基-苯并 $[d]$ 咪唑-2-基)乙基]-1-丁胺(1b): 粘稠液体 $0.58 \mathrm{~g}$, 收率 $17 \% .{ }^{1} \mathrm{H}$ NMR $\left(400 \mathrm{MHz}, \mathrm{CDCl}_{3}\right)$ $\delta: 7.80 \sim 7.78(\mathrm{~m}, 1 \mathrm{H}), 7.49 \sim 7.29(\mathrm{~m}, 3 \mathrm{H}), 4.96(\mathrm{q}, J=$ $6.8 \mathrm{~Hz}, 1 \mathrm{H}), 4.50 \sim 4.42(\mathrm{~m}, 1 \mathrm{H}), 4.28 \sim 4.08(\mathrm{~m}, 1 \mathrm{H})$, $3.27 \sim 3.23(\mathrm{~m}, 1 \mathrm{H}), 3.04 \sim 2.86(\mathrm{~m}, 1 \mathrm{H}), 1.99(\mathrm{~d}, J=7.8$ $\mathrm{Hz}, 3 \mathrm{H}), 1.93 \sim 1.90(\mathrm{~m}, 2 \mathrm{H}), 1.86 \sim 1.72(\mathrm{~m}, 2 \mathrm{H}), 1.52 \sim$ 1.24 (m, 4H), 0.98 (t, $J=5.2 \mathrm{~Hz}, 3 \mathrm{H}), 0.90$ (t, $J=7.4 \mathrm{~Hz}$, $3 \mathrm{H}) ;{ }^{13} \mathrm{C}$ NMR (101 MHz, $\left.\mathrm{CDCl}_{3}\right) \delta: 149.3,141.8,134.9$, 123.7, 123.0, 120.0, 110.4, 50.5, 45.9, 44.3, 32.3, 28.6, 20.2, 19.9, 19.2, 13.7, 13.4; IR (KBr) v: 3441, 2959, 2933, 2871, 2782, 1884, 1764, 1614, 1561, 1515, 1471, 1457, $1379,1280,1093,746 \mathrm{~cm}^{-1}$. Anal. calcd for $\mathrm{C}_{17} \mathrm{H}_{27} \mathrm{~N}_{3}$ : C 74.68, H 9.95, N 15.37; found C 74.75, H 9.78, N 15.45.

$N$-[1-(1H-苯并 $[d]$ 咪唑-2-基)乙基]- $N$-丁基-1-丁胺 (1c): 粘稠液体 $0.33 \mathrm{~g}$, 收率 $13 \% .{ }^{1} \mathrm{H}$ NMR $(400 \mathrm{MHz}$, $\left.\mathrm{CDCl}_{3}\right) \delta: 7.76 \sim 7.67(\mathrm{~m}, 1 \mathrm{H}), 7.44 \sim 7.42(\mathrm{~m}, 1 \mathrm{H})$, $7.25 \sim 7.17(\mathrm{~m}, 2 \mathrm{H}), 4.21(\mathrm{q}, J=5.6 \mathrm{~Hz}, 1 \mathrm{H}), 2.51 \sim 2.40$ (m, $4 \mathrm{H}), 1.65 \sim 1.54(\mathrm{~m}, 7 \mathrm{H}), 1.48 \sim 1.25(\mathrm{~m}, 4 \mathrm{H}), 1.02 \sim$ $0.83(\mathrm{~m}, 6 \mathrm{H}) ;{ }^{13} \mathrm{C}$ NMR $\left(101 \mathrm{MHz}, \mathrm{CDCl}_{3}\right) \delta: 158.8$, 143.8, 133.2, 122.5, 121.7, 119.4, 110.4, 54.6, 50.2, 30.77, 20.62, 14.06, 10.39; IR (KBr) v: 3469, 2954, 2931, 2867, 2819, 2730, 1913, 1877, 1620, 1455, 1415, 1367, 1314, 1273, 1186, 1107, 1096, $743 \mathrm{~cm}^{-1}$. Anal. calcd for $\mathrm{C}_{17} \mathrm{H}_{27} \mathrm{~N}_{3}$ : C 74.68, H 9.95, N 15.37; found C 74.70, H 9.81, N 15.48 .

$N$-[1-(1-丁基-苯并 [ $d$ ]咪唑-2-基)乙基]- $N$-丁基-1-丁 胺(1d): 粘稠液体 $0.69 \mathrm{~g}$, 收率 $22 \% .{ }^{1} \mathrm{H}$ NMR $(400 \mathrm{MHz}$, $\left.\mathrm{CDCl}_{3}\right) \delta: 7.79 \sim 7.74(\mathrm{~m}, 1 \mathrm{H}), 7.34 \sim 7.32(\mathrm{~m}, 1 \mathrm{H})$, $7.32 \sim 7.21(\mathrm{~m}, 2 \mathrm{H}), 4.31 \sim 4.30(\mathrm{~m}, 1 \mathrm{H}), 4.29 \sim 4.25(\mathrm{~m}$, $1 \mathrm{H}), 4.18 \sim 4.02(\mathrm{~m}, 1 \mathrm{H}), 2.62 \sim 2.47(\mathrm{~m}, 2 \mathrm{H}), 2.47 \sim 2.34$ $(\mathrm{m}, 2 \mathrm{H}), 1.92 \sim 1.68(\mathrm{~m}, 2 \mathrm{H}), 1.55(\mathrm{~d}, J=7.2 \mathrm{~Hz}, 3 \mathrm{H})$, $1.49 \sim 1.33(\mathrm{~m}, 4 \mathrm{H}), 1.35 \sim 1.06(\mathrm{~m}, 6 \mathrm{H}), 0.96(\mathrm{t}, J=8.7$ $\mathrm{Hz}, 3 \mathrm{H}), 0.80(\mathrm{t}, J=7.3 \mathrm{~Hz}, 6 \mathrm{H}) ;{ }^{13} \mathrm{C}$ NMR $(101 \mathrm{MHz}$, $\left.\mathrm{CDCl}_{3}\right) \delta: 155.5,142.2,135.7,122.1,121.4,119.6,109.4$, 53.2, 50.1, 43.4, 32.1, 30.9, 20.5, 13.9, 9.9; IR (KBr) v: 2957, 2871, 2861, 1690, 1614, 1503, 1459, 1412, 1367, $1277,1091,737 \mathrm{~cm}^{-1}$. Anal. calcd for $\mathrm{C}_{21} \mathrm{H}_{35} \mathrm{~N}_{3}: \mathrm{C} 76.54$, H 10.71, N 12.75; found C 76.60, H 10.78, N 12.62 .

\section{4 叔丁基-1-(1H-苯并 [d]咪唑-2-基)氨基甲酸乙酯 (1e)的合成}

$100 \mathrm{~mL}$ 三口瓶中加入 $\mathrm{NaOH} 0.48 \mathrm{~g}(0.012 \mathrm{~mol}), \mathbf{1}$

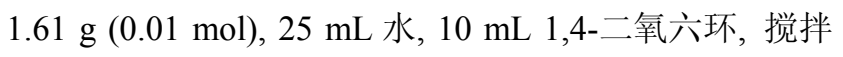
均匀后, 于冰浴下滴加溶有 $\mathrm{Boc}_{2} \mathrm{O} 2.62 \mathrm{~g}(0.012 \mathrm{~mol})$ 的
1,4-二氧六环溶液 $15 \mathrm{~mL}$, 滴毕室温反应. TLC 检测反应 进程, 反应过程中有大量白色固体生成. 反应毕, 过滤, 滤饼经水洗三次, 石油醚洗两次, 干燥后得白色固体 2.14 g, 收率 82\%. m.p. $183 \sim 185{ }^{\circ} \mathrm{C} ;{ }^{1} \mathrm{H}$ NMR (400 $\left.\mathrm{MHz}, \mathrm{CDCl}_{3}\right) \delta: 7.60 \sim 7.46(\mathrm{~m}, 2 \mathrm{H}), 7.25 \sim 7.22(\mathrm{~m}, 2 \mathrm{H})$, $5.27(\mathrm{~s}, 1 \mathrm{H}), 5.02(\mathrm{q}, J=7.7 \mathrm{~Hz}, 1 \mathrm{H}), 1.75(\mathrm{~d}, J=7.2 \mathrm{~Hz}$, $3 \mathrm{H}), 1.46(\mathrm{~s}, 9 \mathrm{H}) ;{ }^{13} \mathrm{C}$ NMR (101 MHz, DMSO- $\left.d_{6}\right) \delta$ : 156.4, 155.0, 121.2, 78.1, 45.0, 28.2, 20.1; IR (KBr) v: 3329, 2981, 2875, 2738, 1680, 1527, 1445, 1384, 1324, $1264,1171,1069,994,863,743,629 \mathrm{~cm}^{-1}$. Anal. calcd for $\mathrm{C}_{14} \mathrm{H}_{19} \mathrm{~N}_{3} \mathrm{O}_{2}$ : C 64.35, H 7.33, N 16.08; found C 64.41, H 7.21, N 15.96 .

\section{5 叔丁基-1-(1-丁基-苯并 [ $d]$ 咪唑-2-基)氨基甲酸乙 酯(1f)的合成}

合成方法同第 1.3 节. 将 1 e $0.26 \mathrm{~g}(1 \mathrm{mmol}), \mathrm{KOH}$ $0.12 \mathrm{~g}(2 \mathrm{mmol})$ 和 $30 \mathrm{~mL}$ 异丙醇常温搅拌 $0.5 \mathrm{~h}$ 后加入 溴丁烷 $0.21 \mathrm{~g}(1.5 \mathrm{mmol})$, 加热至回流反应, 产物经柱 层析分离纯化 [淋洗剂: $V$ (乙酸乙酯) $: V$ (石油醚) $=1$ : 2], 最后得到白色固体 $0.24 \mathrm{~g}$, 收率 75\%. m.p. 125 126 ${ }^{\circ} \mathrm{C} ;{ }^{1} \mathrm{H}$ NMR (400 MHz, $\left.\mathrm{CDCl}_{3}\right) \delta: 7.76 \sim 7.72(\mathrm{~m}, 1 \mathrm{H})$, $7.37 \sim 7.35(\mathrm{~m}, 1 \mathrm{H}), 7.30 \sim 7.25(\mathrm{~m}, 2 \mathrm{H}), 5.45(\mathrm{~s}, 1 \mathrm{H})$, $5.20 \sim 5.13(\mathrm{~m}, 1 \mathrm{H}), 4.32 \sim 4.25(\mathrm{~m}, 1 \mathrm{H}), 4.19 \sim 4.12(\mathrm{~m}$, $1 \mathrm{H}), 1.81(\mathrm{dt}, J=15.3,7.6 \mathrm{~Hz}, 2 \mathrm{H}), 1.66(\mathrm{~d}, J=6.8 \mathrm{~Hz}$, $3 \mathrm{H}), 1.43 \sim 1.39(\mathrm{~m}, 11 \mathrm{H}), 0.97(\mathrm{t}, J=7.4 \mathrm{~Hz}, 3 \mathrm{H}) ;{ }^{13} \mathrm{C}$ NMR $\left(101 \mathrm{MHz}, \mathrm{CDCl}_{3}\right) \delta: 155.2,154.9,142.2,134.7$, 122.5, 122.0, 119.2, 109.5, 79.3, 43.4, 42.5, 32.0, 28.4, 21.1, 19.9, 13.3; IR (KBr) v: 3356, 3337, 2983, 2964, 2932, 2868, 1681, 1518, 1468, 1371, 1334, 1247, 1174, $1061,876,741 \mathrm{~cm}^{-1}$. Anal. calcd for $\mathrm{C}_{18} \mathrm{H}_{27} \mathrm{~N}_{3} \mathrm{O}_{2}: \mathrm{C} 68.11$, H 8.57, N 13.24; found C 68.31, H 8.63, N 13.26.

\subsection{1-(1-丁基-苯并[ $d$ ]咪唑-2-基)乙胺(1g)的合成}

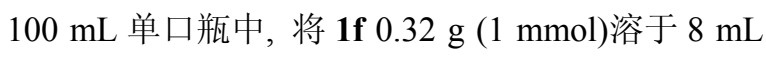
乙酸乙酯溶液中, 冰浴下加入 $0.9 \mathrm{~mL} \mathrm{HCl}$ (约 $10 \mathrm{mmol}$ ), TLC 检测反应进程. $4 \mathrm{~h}$ 后反应毕, 旋蒸除去乙酸乙酯及 过量的 $\mathrm{HCl}$, 用 $\mathrm{Na}_{2} \mathrm{CO}_{3}$ 溶液调体系的 $\mathrm{pH}$ 至 $8 \sim 9$, 旋蒸 除去水, 得到的白色固体用 $\mathrm{CHCl}_{3}$ 溶液洗, 过滤, 滤液 浓缩, 得到淡黄色粘稠液 $0.18 \mathrm{~g}$, 收率 $82 \% .{ }^{1} \mathrm{H}$ NMR $\left(400 \mathrm{MHz}, \mathrm{CDCl}_{3}\right) \delta: 7.74 \sim 7.73(\mathrm{~m}, 1 \mathrm{H}), 7.33 \sim 7.31(\mathrm{~m}$, $1 \mathrm{H}), 7.26 \sim 7.23(\mathrm{~m}, 2 \mathrm{H}), 4.33 \sim 4.31(\mathrm{~m}, 1 \mathrm{H}), 4.21 \sim 4.12$ $(\mathrm{m}, 2 \mathrm{H}), 2.36(\mathrm{~s}, 2 \mathrm{H}), 1.83 \sim 1.78(\mathrm{~m}, 2 \mathrm{H}), 1.61(\mathrm{~d}, J=6.4$ $\mathrm{Hz}, 3 \mathrm{H}), 1.44 \sim 1.39(\mathrm{~m}, 2 \mathrm{H}), 0.97(\mathrm{t}, J=7.4 \mathrm{~Hz}, 3 \mathrm{H}) ;{ }^{13} \mathrm{C}$ NMR (101 MHz, $\left.\mathrm{CDCl}_{3}\right) \delta: 158.3,142.3,135.1,122.3$, 121.9, 119.4, 109.6, 44.2, 43.5, 32.1, 23.9, 20.2, 13.7; IR (KBr) v: 3356, 3286, 3053, 2961, 2872, 1613, 1502, 1459, $1424,1330,1281,1065,952,747 \mathrm{~cm}^{-1}$. Anal. calcd for 
$\mathrm{C}_{13} \mathrm{H}_{19} \mathrm{~N}_{3}$ : C 71.85, H 8.81, N 19.34; found $\mathrm{C} 72.10, \mathrm{H}$ $8.73, \mathrm{~N} 19.16$.

\section{7 叔丁基-1-(1-苄基-苯并[ $d$ ]咪唑-2-基)氨基甲酸乙 酯(1h)的合成}

合成方法同第 1.3 节, 将 1 e $0.26 \mathrm{~g}$ (1 mmol), $\mathrm{KOH}$ $0.12 \mathrm{~g}(2 \mathrm{mmol})$ 和 $30 \mathrm{~mL}$ 异丙醇常温摚拌 $0.5 \mathrm{~h}$ 后加入氯 化苠 $0.19 \mathrm{~g}$ (1.5 mmol), 产物经柱层析分离纯化 [淋洗剂: $V($ 乙酸乙酯 $): V($ 石油醚 $)=1 ： 3$, 最后得到白色固体 0.26 g, 收率 74\%. m.p. 174 $176{ }^{\circ} \mathrm{C} ;{ }^{1} \mathrm{H}$ NMR (400 $\left.\mathrm{MHz}, \mathrm{CDCl}_{3}\right) \delta: 7.80 \sim 7.78(\mathrm{~m}, 1 \mathrm{H}), 7.32 \sim 7.27(\mathrm{~m}, 5 \mathrm{H})$, $7.09 \sim 7.07(\mathrm{~m}, 2 \mathrm{H}), 5.56 \sim 5.46(\mathrm{~m}, 2 \mathrm{H}), 5.35(\mathrm{~s}, 1 \mathrm{H})$, $5.16 \sim 5.12(\mathrm{~m}, 1 \mathrm{H}), 1.57(\mathrm{~d}, J=6.8 \mathrm{~Hz}, 3 \mathrm{H}), 1.38(\mathrm{~s}, 9 \mathrm{H})$; ${ }^{13} \mathrm{C}$ NMR (101 MHz, $\left.\mathrm{CDCl}_{3}\right) \delta: 155.7,154.9,142.3,136.1$, $135.3,128.9,127.8,126.3,123.0,122.4,119.7,110.2$, 79.8, 46.9, 42.8, 28.3, 20.9; IR (KBr) v: 3341, 3063, 3034, 2981, 2932, 1677, 1524, 1455, 1411, 1332, 1250, 1172, 1064, 751, $724 \mathrm{~cm}^{-1}$. Anal. calcd for $\mathrm{C}_{21} \mathrm{H}_{25} \mathrm{~N}_{3} \mathrm{O}_{2}: \mathrm{C} 71.77$, H 7.17, N 11.96; found C 71.98, H 7.33, N 12.16.

\subsection{1-(1-苄基-苯并 $[d]$ 咪唑-2-基)乙胺(1i)的合成}

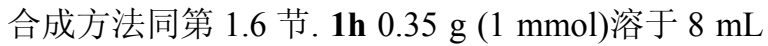
乙酸乙酯溶液中, 冰浴下加入 $0.9 \mathrm{~mL} \mathrm{HCl}$ (约 $10 \mathrm{mmol})$, 反应毕经纯化得淡黄色粘稠液体 $0.22 \mathrm{~g}$, 收率 $88 \%$. ${ }^{1} \mathrm{H}$ NMR $\left(400 \mathrm{MHz}, \mathrm{CDCl}_{3}\right) \delta: 7.97 \sim 7.22(\mathrm{~m}, 1 \mathrm{H}), 7.46 \sim$ $7.16(\mathrm{~m}, 6 \mathrm{H}), 7.12 \sim 6.96(\mathrm{~m}, 2 \mathrm{H}), 5.47(\mathrm{q}, J=16.8 \mathrm{~Hz}$, 2H), 4.29 (q, $J=6.7 \mathrm{~Hz}, 1 \mathrm{H}), 2.49$ (s, 2H), 1.53 (d, $J=6.7$ $\mathrm{Hz}, 3 \mathrm{H}) ;{ }^{13} \mathrm{C} \mathrm{NMR}\left(101 \mathrm{MHz}, \mathrm{CDCl}_{3}\right) \delta: 174.6,158.5$, $142.2,136.1,135.6,129.0,128.8,127.9,126.1,125.8$, 122.8, 122.3, 119.8, 109.8, 46.8, 44.5, 23.5; IR (KBr) $v$ : 3355, 3277, 3053, 2974, 2926, 1502, 1457, 1417, 738 $\mathrm{cm}^{-1}$. Anal. calcd for $\mathrm{C}_{16} \mathrm{H}_{17} \mathrm{~N}_{3}$ : C 76.46, H 6.82, N 16.72; found C 76.24, H 7.09, N 16.66.

\section{9 苯乙酮的氢转移反应}

在氮气保护下，向 $100 \mathrm{~mL}$ 厚壁 Schlenk 管中加入一 定量的催化剂前体、配体、苯乙酮、异丙醇钠的异丙醇 溶液及计算量的异丙醇，混合体系经抽、排 3 次后在氮 气下封闭, 在一定温度下, 反应一定时间后冷却, 溶液 经硅胶柱过滤, 脱除金属化合物后气相色谱 (CP-Chirasil-Dex CB) 检测分析，计算催化效率.

\section{2 结果与讨论}

\section{1 氨基取代苯并咪唑的合成}

邻苯二胺和氨基酸的反应已有文献报道, 不同文献 描述的不尽相同. 当用 $6 \mathrm{~mol} \cdot \mathrm{L}^{-1}$ 盐酸做催化剂时, 反应 很慢, 需要很长时间回流反应 ${ }^{[9,12]}$. 刘思全等 ${ }^{[13]}$ 报道用
磷酸、 PPA(多聚磷酸)在 $200{ }^{\circ} \mathrm{C}$ 下催化该反应取得很好 的结果. 在我们实验中, $6 \mathrm{~mol} \cdot \mathrm{L}^{-1}$ 盐酸催化邻苯二胺和 丙氨酸的反应很慢，加热回流 $72 \mathrm{~h}$ 后薄层检测显示仍有 大量未反应的原料; 以乙二醇为溶剂丙氨酸盐酸盐与邻 苯二胺反应可以形成产物，但产物和盐分离较困难，采 用磷酸和 PPA 混酸作溶剂及催化剂，上述反应在 180 $200{ }^{\circ} \mathrm{C}$ 需要 $6 \sim 8 \mathrm{~h}$ 才能反应完全. 张精安等 ${ }^{[14]}$ 采用微 波辅助、 $6 \mathrm{~mol} \cdot \mathrm{L}^{-1}$ 盐酸催化氨基乙酸与邻苯二胺反应合 成 2-氨甲基苯并咪唑. 为此，我们考察了邻苯二胺与氨 基酸的物质的量比 $1: 1$, 磷酸 $: P P A(V: V=2.5: 1)$ 为 催化剂, 经微波辐照合成苯并咪唑衍生物. 研究发现氨 基酸与邻苯二胺的缩合反应在微波辐照下 $0.5 \mathrm{~h}$ 就基本 完全. 氨基取代苯并咪唑的合成反应式见 Scheme 1. 所 得化合物经 NMR，FT-IR 及元素分析确认．旋光测定发 现，产物氨基取代的苯并咪唑发生了外消旋化. 氨基酸 在酸、碱、高温及微波辐照下可以发生消旋化反应 ${ }^{[15]}$. 手性氨基取代的苯并咪唑的合成有文献报道 ${ }^{[6]}$, 在该条 件下反应很慢, 转化率很低. 酸性条件下的外消旋化的 可能途径见 Scheme 2, 邻苯二胺与氨基酸首先形成酰胺 $\mathbf{A}$, 氨基进一步与酰胺的 $\mathrm{C}=\mathrm{O}$ 加成形成中间体 $\mathbf{B}, \mathbf{B}$ 脱 水可以形成中间体 $\mathbf{C}$ 和 $\mathbf{D}, \mathbf{D}$ 经 1,3-氢迁移可形成外消 旋化的胺.
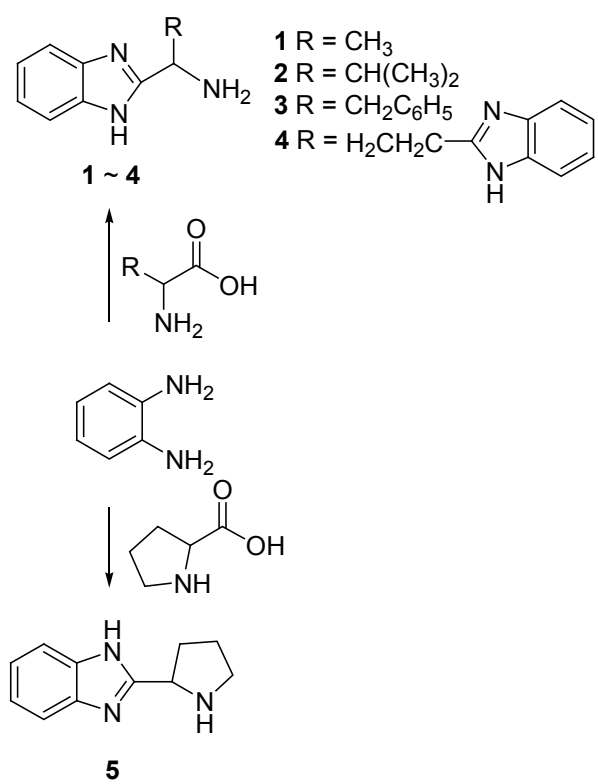

Scheme 1

氨基取代苯并咪唑分子中有 3 个氮原子，其衍生物 可用于研究取代基效应. 1-(1H-苯并 $[d]$ 咪唑-2-基)乙胺 (1)与溴丁烷直接反应，反应选择性差，经柱层析分离得 到 4 种物质 $1 \mathbf{a} \sim \mathbf{1 d}$ ，化合物 1 中伯氨基反应活性高，先 形成单取代的 $1 \mathrm{a}$, 此时 $1 \mathrm{a}$ 分子中含有二个仲胺官能团, 可以进一步 $N$-烷基化, 形成 1b 及 1c, 1b 及 $1 \mathrm{c}$ 进一步 $N$-烷基化形成 1d. 该反应虽然选择性差，但一次合成出 


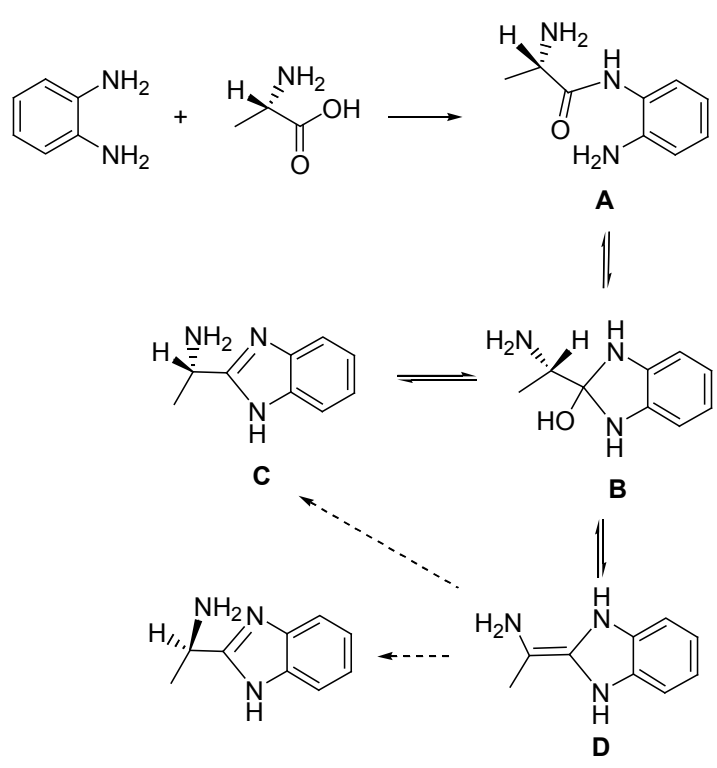

Scheme 2

不同结构的四个化合物, 方便了咪唑衍生物催化性能研 究的需要. 为了进一步研究咪唑衍生物的取代基对其催 化性能的影响, 先将 $\mathbf{1}$ 中伯氨基用 Boc 保护, 再与卤代 烃反应, 即可得到咪唑环上单取代的产物 $1 \mathrm{f}$ 和 $1 \mathrm{~h}$, 脱除 Boc 保护基团得到 $1 \mathrm{~g}$ 和 1ii，反应式见 Scheme 3.

正丁基取代的苯并咪唑类产物的结构经核磁分析 (图 1), 从图中可以看出由于苯并咪唑环共轭效应的影 响, 环上与 $\mathrm{N}$ 相连丁基中的亚甲基处于低场, 化学位移
较大 $(\mathbf{1 b}, 1 \mathrm{~d}, 1 \mathrm{~g})$, 化合物的结构得到 NMR, FT-IR 及元 素分析的验证.

\section{2 催化性能的研究}

在研究催化性能的初期, 我们考察了配体 1, 2 与 $\left[\mathrm{RuCl}_{2}(p \text {-Cymene })\right]_{2}, \mathrm{RuCl}_{2}\left(\mathrm{PPh}_{3}\right)_{3}, \mathrm{RuCl}_{3}$ 络合物催化体 系对苯乙酮氢转移反应的催化性能，苯乙酮选择性被还 原成 2-苯乙醇, 结果列于表 1 . 从表中可以看出 $25{ }^{\circ} \mathrm{C}$ 时, 只有 $1 /\left[\mathrm{RuCl}_{2}(p \text {-Cymene) }]_{2}\right.$ 催化体系苯乙酮的转化 率达到 94\%, 配体 1, 2 与各催化剂前体组成的催化体系 几乎没有催化活性; $90{ }^{\circ} \mathrm{C}$ 时, 各催化体系的催化活性都 有了一定程度的提高, 以 $\left[\mathrm{RuCl}_{2}(p \text {-Cymene })\right]_{2}$, $\mathrm{RuCl}_{2}\left(\mathrm{PPh}_{3}\right)_{3}$ 的催化活性较好, $3 \mathrm{~h}$ 时苯乙酮的转化率都 已经达到了 $95 \%$ 以上, 而以 $\mathrm{RuCl}_{3}$ 为催化剂前体的催化 剂活性只有 $40 \%$ 左右.

不同氨基取代苯并咪唑与 $\mathrm{RuCl}_{2}\left(\mathrm{PPh}_{3}\right)_{3}$ 组成的催化 体系对苯乙酮氢转移反应的结果列于表 2. 从表 2 中可 以看出, 咪唑环上引入取代基有利于提高形成钓催化剂 的催化活性, 1e, 1f, 1g, 1i 参与反应的催化活性较高. 在 相同的催化条件下, 氨基取代咪坐 $\mathbf{1} \sim \mathbf{5}$ 与 $\mathrm{RuCl}_{2}\left(\mathrm{PPh}_{3}\right)_{3}$ 的催化活性随时间的关系如图 2 所示. 从图 2 中可以看 出, $2 / \mathrm{RuCl}_{2}\left(\mathrm{PPh}_{3}\right)_{3}$ 催化体系苯乙酩的转化率迅速上升, 很快趋于稳定, $40 \mathrm{~min}$ 时转化率已经达到 $91 \%, \mathbf{1}, 5$ 与 $\mathrm{RuCl}_{2}\left(\mathrm{PPh}_{3}\right)_{3}$ 的催化体系的苯乙酮转化率上升的相对慢<smiles>CCCCBr</smiles><smiles>CCCCNC(C)c1nc2ccccc2[nH]1</smiles>

1a<smiles>CCCCNC(C)c1nc2ccccc2n1CCCC</smiles>

$1 b$

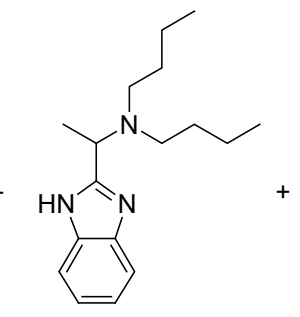

1c<smiles>CCCCN(CCCC)C(C)c1nc2ccccc2n1CCCC</smiles>

1d<smiles>CCCCC(C)C(=O)NC(C)c1nc2ccccc2[nH]1</smiles>

$1 e$<smiles>CCCCn1c(C(C)NC(=O)OC(C)(C)C)nc2ccccc21</smiles>

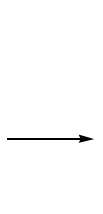

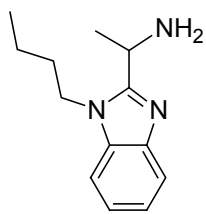

$1 \mathrm{~g}$<smiles>CC(NC(=O)OC(C)(C)C)c1nc2ccccc2n1Cc1ccccc1</smiles>

1h<smiles>CC(N)c1nc2ccccc2n1Cc1ccccc1</smiles>

$1 \mathrm{i}$

Scheme 3 


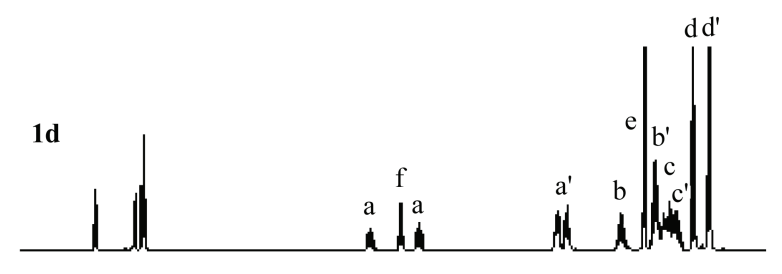

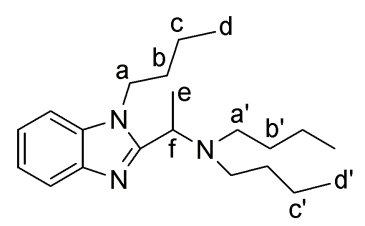
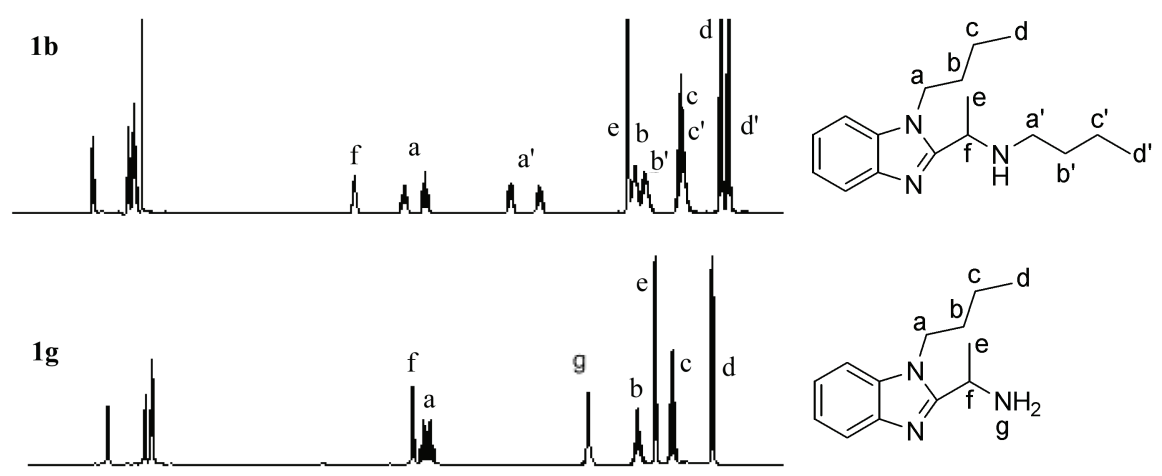

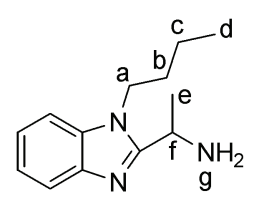
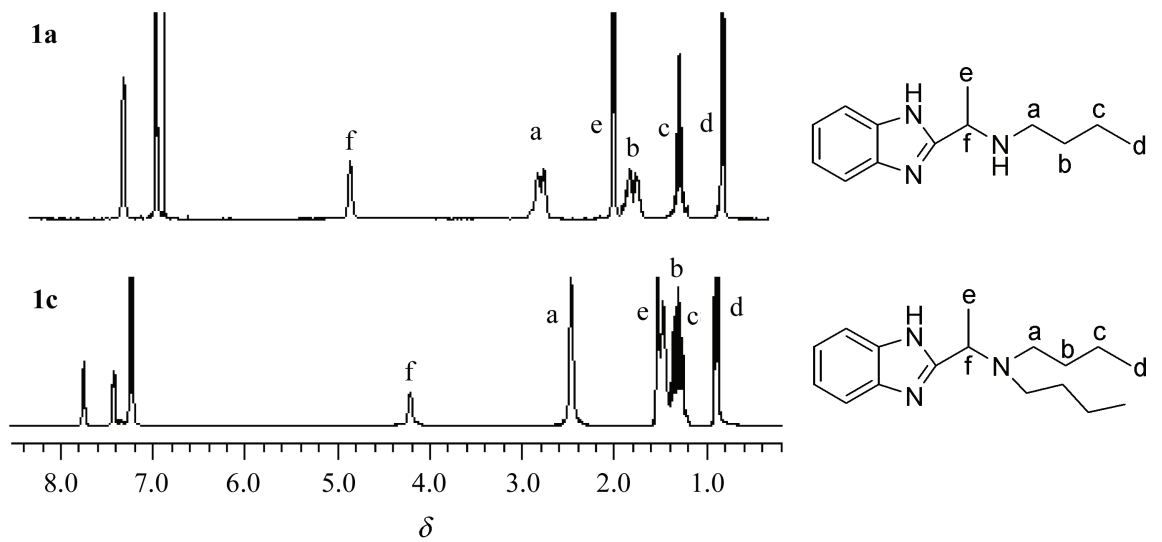

图 1 氨基取代苯并咪唑的核磁谱图

Figure $1{ }^{1} \mathrm{H}$ NMR spectra of aminated benzimidazoles

表 1 配体 1,2 与钉前体络合物催化的苯乙酮氢转移反应 ${ }^{a}$

Table 1 Transfer hydrogenation of acetophenone promoted by complexes of $\mathbf{1}, \mathbf{2}$ and $\mathrm{Ru}$ precursors<smiles></smiles>

\begin{tabular}{cclcc}
\hline Entry & 配体 & \multicolumn{1}{c}{ 催化剂前体 } & $t{ }^{\circ} \mathrm{C}$ & 转化率 $/ \%$ \\
\hline 1 & 1 & {$\left[\mathrm{RuCl}_{2}(p-\mathrm{Cymene})\right]_{2}$} & 25 & 94 \\
2 & 1 & $\mathrm{RuCl}_{2}\left(\mathrm{PPh}_{3}\right)_{3}$ & 25 & 1.3 \\
3 & 1 & $\mathrm{RuCl}_{3}$ & 25 & 0 \\
4 & 2 & {$\left[\mathrm{RuCl}_{2}(p-\mathrm{Cymene})\right]_{2}$} & 25 & 0 \\
5 & 2 & $\mathrm{RuCl}_{2}\left(\mathrm{PPh}_{3}\right)_{3}$ & 25 & 0 \\
6 & 2 & $\mathrm{RuCl}_{3}$ & 25 & 0 \\
7 & 1 & $\mathrm{RuCl}_{2}\left(\mathrm{PPh}_{3}\right)_{3}$ & 90 & 95 \\
8 & 1 & $\mathrm{RuCl}_{3}$ & 90 & 40 \\
9 & 2 & {$\left[\mathrm{RuCl}_{2}\left(p-\mathrm{Cymene}_{2}\right)\right]_{2}$} & 90 & 97 \\
10 & 2 & $\mathrm{RuCl}_{2}\left(\mathrm{PPh}_{3}\right)_{3}$ & 90 & 97 \\
11 & 2 & $\mathrm{RuCl}_{3}$ & 90 & 38 \\
\hline
\end{tabular}

${ }^{a} \mathrm{Ru}(\mathrm{II})$ 催化剂 $0.01 \mathrm{mmol}$, [酮] :[Ru] :[配体]：[iso-PrONa] $=200: 1: 1:$ 10 , 苯乙酮 $0.2 \mathrm{~mol} \cdot \mathrm{L}^{-1}$, 反应 $3 \mathrm{~h}$.
一些，最后也趋于稳定, 3,4 与 $\mathrm{RuCl}_{2}\left(\mathrm{PPh}_{3}\right)_{3}$ 的催化体系 的苯乙酮的转化率随时间几乎线性增加, 催化体系的稳 定性较好. 1b 1i 与 $\mathrm{RuCl}_{2}\left(\mathrm{PPh}_{3}\right)_{3}$ 的催化体系的苯乙酮 转化率与反应时间的曲线见图 3. 从图 3 中看出, 含有 $\mathrm{NH}_{2}$ 官能团的 $1 \mathbf{g}$ 和 $\mathbf{1 i}$ 参与反应催化剂的催化活性最高, 反应 $30 \mathrm{~min}$ 时苯乙酮的转化率已经达到 $90 \%$ 左右, TOF 为 $3600 \mathrm{~h}^{-1}$. 从 $\mathbf{1 b}, \mathbf{1 d}, \mathbf{1 f}, \mathbf{1 g}$ 催化体系的催化活性对比 中可以发现，随着端氨基上的取代基增加，参与反应的 配体催化活性降低, Boc 保护的化合物 $1 \mathbf{f}$ 活性相比最低.

为了进一步考察催化体系的催化性能，反应条件对 $\mathrm{RuCl}_{2}\left(\mathrm{PPh}_{3}\right)_{3} / \mathbf{1 g}$ 催化苯乙酮氢转移反应的反应结果列于 表 3. 从表 3 中可以看出, 催化体系的催化活性随温度、 碱浓度及底物浓度的增大而增加. 碱浓度增大两倍, 苯 乙酩的转化率从 $65 \%$ 增大到 $86 \%$ (表 3, Entries 4, 8); 苯 乙酮的浓度从 $0.2 \mathrm{~mol} \cdot \mathrm{L}^{-1}$ 增大到 $0.8 \mathrm{~mol} \cdot \mathrm{L}^{-1}$ 时苯乙酮 的转化率能从 $65 \%$ 增大到 $89 \%$ (表 3, Entries 4 6), 进一 步增加苯乙酮的浓度到 $1.0 \mathrm{~mol} \cdot \mathrm{L}^{-1}$ 时, 苯乙酮的转化率 
表 $2 \mathrm{RuCl}_{2}\left(\mathrm{PPh}_{3}\right)_{3} / \mathbf{1} \sim \mathbf{1 i}$ 催化的苯乙酮氢转移反应

Table 2 Transfer hydrogenation of acetophenone catalyzed by $\mathrm{RuCl}_{2}\left(\mathrm{PPh}_{3}\right)_{3} / \mathbf{1} \sim \mathbf{1 i}$

\begin{tabular}{ccc||ccc}
\hline Entry & 配体 & 转化率/\% & Entry & 配体 & 转化率/\% \\
\hline 1 & $\mathbf{1}$ & 46 & 8 & $\mathbf{1 c}$ & 52 \\
2 & $\mathbf{2}$ & 63 & 9 & $\mathbf{1 d}$ & 52 \\
3 & $\mathbf{3}$ & 30 & 10 & $\mathbf{1 e}$ & 96 \\
4 & $\mathbf{4}$ & 21 & 11 & $\mathbf{1 f}$ & 91 \\
5 & $\mathbf{5}$ & 46 & 12 & $\mathbf{1 g}$ & 97 \\
6 & $\mathbf{1 a}$ & 60 & 13 & $\mathbf{1 h}$ & 69 \\
7 & $\mathbf{1 b}$ & 73 & 14 & $\mathbf{1 i}$ & 97
\end{tabular}

${ }^{a} \mathrm{RuCl}_{2}\left(\mathrm{PPh}_{3}\right)_{3}$ 催化剂 $0.01 \mathrm{mmol}$, [酮] : [Ru] : [配体] : [iso-PrONa]为 200 : $1: 1: 10$, 苯乙酮 $0.2 \mathrm{~mol} \cdot \mathrm{L}^{-1}, 90{ }^{\circ} \mathrm{C}$ 反应 $20 \mathrm{~min}$

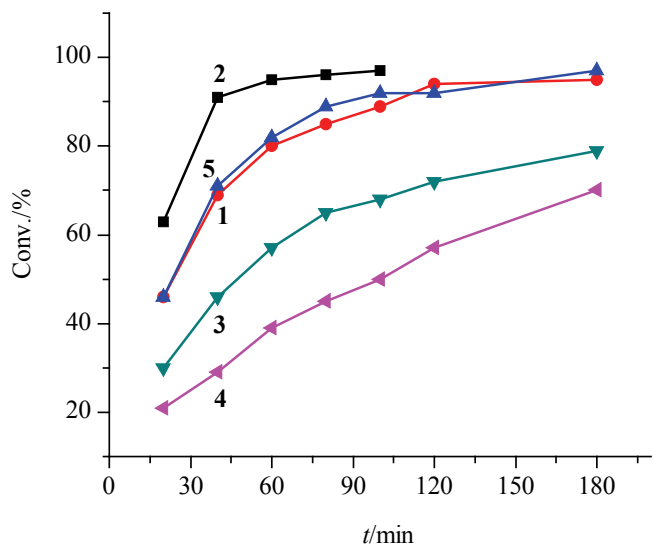

图 $2 \mathrm{RuCl}_{2}\left(\mathrm{PPh}_{3}\right)_{3} / \mathbf{1} \sim \mathbf{5}$ 催化的苯乙酮氢转移反应

Figure 2 Transfer hydrogenation of acetophenone catalyzed by $\mathrm{RuCl}_{2}\left(\mathrm{PPh}_{3}\right)_{3} / \mathbf{1} \sim \mathbf{5}$

$\mathrm{RuCl}_{2}\left(\mathrm{PPh}_{3}\right)_{3}$ 催化剂 $0.01 \mathrm{mmol}$, [酮]：[Ru]：[配体]：[iso-PrONa]= $200: 1: 1: 10$, 苯乙酮 $0.2 \mathrm{~mol} \cdot \mathrm{L}^{-1}, 90{ }^{\circ} \mathrm{C}$ 反应 $3 \mathrm{~h}$

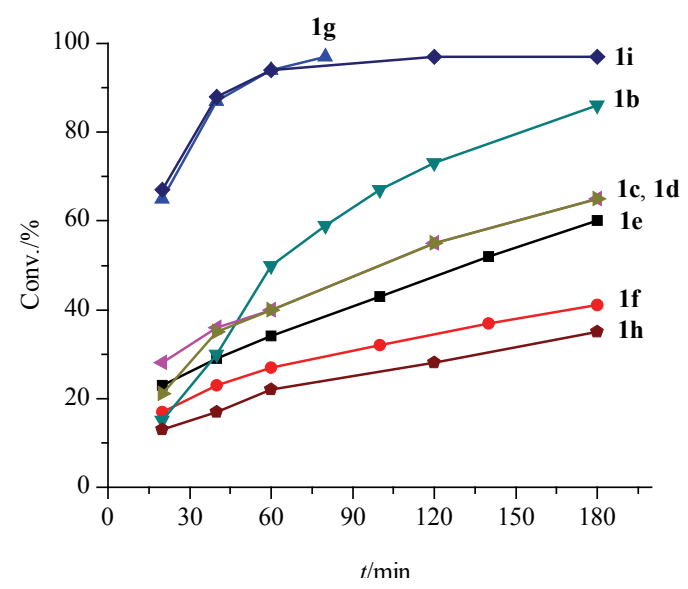

图 $3 \mathrm{RuCl}_{2}\left(\mathrm{PPh}_{3}\right)_{3} / \mathbf{1 b} \sim \mathbf{1 i}$ 催化的苯乙酮氢转移反应

Figure 3 Transfer hydrogenation of acetophenone catalyzed by $\mathrm{RuCl}_{2}\left(\mathrm{PPh}_{3}\right)_{3} / \mathbf{1 b} \sim \mathbf{1 i}$

[酮]：[Ru]：[配体]：[iso-PrONa]为 $2000 ： 1 ： 1 ： 10$, 苯乙酮 0.2 $\mathrm{mol} \cdot \mathrm{L}^{-1}, 110{ }^{\circ} \mathrm{C}$

反而降低到 $85 \%$ (表 3 , Entry 7), 温度升到 $140{ }^{\circ} \mathrm{C}$ 反应 15 $\mathrm{min}$, 苯乙酮的转化率可达到 $97 \%$, TOF 为 $7760 \mathrm{~h}^{-1}$ (表
3, Entry 9); 进一步增加底物浓度、底物与催化剂活性中 心钉的物质的量比, TOF 可达到了 $40200 \mathrm{~h}^{-1}$ (表 3, Entry 13).

表 $3 \mathrm{RuCl}_{2}\left(\mathrm{PPh}_{3}\right)_{3} / \mathbf{1 g}$ 催化条件的优化 ${ }^{a}$

Table 3 Optimization reaction condition of $\mathrm{RuCl}_{2}\left(\mathrm{PPh}_{3}\right)_{3} / \mathbf{1 g}$

\begin{tabular}{|c|c|c|c|c|c|}
\hline Entry & $t /{ }^{\circ} \mathrm{C}$ & $\mathrm{S} / \mathrm{C}$ & {$[\mathrm{S}]$} & 转化率/\% & $\mathrm{TOF} / \mathrm{h}^{-1}$ \\
\hline 1 & 90 & 200 & 0.2 & 97 & 582 \\
\hline 2 & 90 & 500 & 0.2 & 83 & 1245 \\
\hline 3 & 110 & 1000 & 0.2 & 97 & 2910 \\
\hline 4 & 110 & 2000 & 0.2 & 65 & 3900 \\
\hline 5 & 110 & 2000 & 0.5 & 75 & 4500 \\
\hline 6 & 110 & 2000 & 0.8 & 89 & 5340 \\
\hline 7 & 110 & 2000 & 1.0 & 85 & 5100 \\
\hline $8^{b}$ & 110 & 2000 & 0.2 & 86 & 5160 \\
\hline $9^{c}$ & 140 & 2000 & 0.2 & 97 & 7760 \\
\hline $10^{b}$ & 140 & 4000 & 0.8 & 91 & 10920 \\
\hline $11^{b}$ & 140 & 5000 & 0.8 & 90 & 13500 \\
\hline $12^{b}$ & 140 & 10000 & 0.8 & 88 & 26400 \\
\hline $13^{b}$ & 140 & 20000 & 0.8 & 67 & 40200 \\
\hline
\end{tabular}

为了拓展催化体系的适用性, $\mathrm{RuCl}_{2}\left(\mathrm{PPh}_{3}\right)_{3} / \mathbf{1 g}$ 对取 代苯乙酮的催化活性列于表 4. 从表中可以看出，取代 苯乙酮的反应活性随对位、间位及邻位依次减低，邻羟 基苯乙酮在该催化体系的催化下没有发生氢转移反应.

表 $4 \mathrm{RuCl}_{2}\left(\mathrm{PPh}_{3}\right)_{3} / \mathbf{1 g}$ 催化芳香酮的氢转移反应 ${ }^{a}$

Table 4 Transfer hydrogenation of aromatic ketones catalyzed by $\mathrm{RuCl}_{2}\left(\mathrm{PPh}_{3}\right)_{3} / \mathbf{1 g}$

\begin{tabular}{|c|c|c|c|}
\hline Entry & 酮 & 转化率/\% & TON \\
\hline 1 & & 50 & 5000 \\
\hline 2 & & 44 & 4400 \\
\hline 3 & & 5 & 500 \\
\hline 4 & & 0 & 0 \\
\hline
\end{tabular}

${ }^{a}$ 酮 $]:[\mathrm{Ru}]:[$ 配体 $]:[$ iso-PrONa $]$ 为 $10000: 1: 1: 20,[\mathrm{~S}]=0.8,140{ }^{\circ} \mathrm{C}$ 反 应 $3 \mathrm{~h}$.

\section{3 结论}

采用微波辐射，以磷酸及多聚磷酸催化氨基酸和邻 苯二胺的无溶剂下反应合成了一系列氨基取代苯并咪 
唑类配体，进一步 $N$-烷基化反应合成不同取代的苯并 咪唑衍生物. 将其与 $\mathrm{Ru}(\mathrm{II})$ 催化剂前体组成催化体系, 考察原位形成的催化剂对取代苯乙酮氢转移反应的催 化活性. $\mathrm{RuCl}_{2}\left(\mathrm{PPh}_{3}\right)_{3}$ 为催化剂前体时, 含伯氨基咪唑环 上 $N$-烷基化的苯并咪唑衍生物参与的催化反应活性高, 催化活性随反应温度、底物浓度增加而增加, $\mathrm{RuCl}_{2}\left(\mathrm{PPh}_{3}\right)_{3} / \mathbf{1 g}$ 催化体系的催化活性 TOF 可达 40200 $\mathrm{h}^{-1}$.

\section{References}

[1] (a) Johnstone, R. A. W.; Wilby, A. H. Chem. Rev. 1985, 85, 129. (b) Zassinovich, G.; Mestroni, G.; Gladiali, S. Chem. Rev. 1992, 92, 1051 .

(c) Palmer, M. J.; Wills, M. Tetrahedron: Asymmetry. 1999, 10, 2045.

[2] (a) Cheng, Z.-B.; Yu, S.-L.; Li, Y.-Y.; Dong, Z.-R.; Sun, G.-S.; Huang, K.-L.; Gao, J.-X. Chem. Res. Chin. Univ. 2011, 27, 170. (b) Ahlford, K.; Ekstrçm, J.; Zaitsev, A. B.; Ryberg, P.; Eriksson, L.; Adolfsson, H. Chem. Eur. J. 2009, 15, 11197.

[3] (a) Frediani, P.; Rosi, L.; Cetarini, L.; Frediani, M. Inorg. Chim. Acta 2006, 359, 2650

(b) Nait Ajjou, A.; Pinet, J. L. J. Mol. Catal. A: Chem. 2004, 214, 203.

[4] (a) Chen, J.; Li, Y.; Dong, Zh.; Li, B.; Gao, J. Tetrahedron Lett. 2004, 45, 8415 .

(b) Li, Y.; Zhang, H.; Chen, J.; Liao, X.; Dong, Z.; Gao, J. J. Mol. Catal. A: Chem. 2004, 218, 153.

(c) Diez, C.; Nagel, U. Appl. Organomet. Chem. 2010, 24, 509.

[5] (a) Arai, N.; Suzuki, K.; Sugizaki, S.; Sorimachi, H.; Ohkuma, T. Angew. Chem., Int. Ed. 2008, 47, 1770.

(b) Baratta, W.; Ballico, M.; Chelucci, G.; Siega, K.; Rigo, P.
Angew. Chem., Int. Ed. 2008, 47, 4362.

[6] Li, Y.; Ding, K.; Sandoval, Ch. A. Org. Lett. 2009, 11, 907.

[7] Li, X.-N.; Zhou, H.-Y.; Feng, L.; Duan, K.; Wang, J.-X. Appl. Organomet. Chem. 2012, 26, 168.

[8] Hallman, P. S.; Stephenson, T. A.; Wilkinson, G. Inorg. Synth. 1970, $12,237$.

[9] Aminabhavi, T. M.; Biradar, N. S.; Patil, S. B. Inorg. Chim. Acta 1986, 125, 125.

[10] Shi, X.-F.; Zhang, M.-J.; Yang, Q.-C.; Cai, G.-M.; Li, J. Chem. Reag. 2001, 23, 355 (in Chinese).

(施秀芳, 张明杰, 杨启超, 蔡桂梅, 李靖, 化学试剂, 2001, 23, 355.)

[11] Chen, G.; Wang, Y.; Yang, X.-S.; Hao, X.-J. Chin. J. Synth. Chem. 2010, 18, 738 (in Chinese). (陈刚, 汪冶, 杨小生, 郝小江, 合成化学, 2010, 18, 738.)

[12] Alatorre-Santamaría, S.; Gotor-Fernández, V.; Gotor, V. Eur. J. Org. Chem. 2009, 2533.

[13] Liu, S.-Q.; Bi, C.-F.; Wang, L.-G. Chin. J. Fine Chem. 2009, 26, 102 (in Chinese)

(刘思全，毕彩丰，王立国，精细化工, 2009, 26, 102.)

[14] Zhang, J.-A.; Bao, J.; Zhang, Z.-Y.; Lin, D.-H. Spec. Petrochem. 2008, 25, 19 (in Chinese).

(张精安, 鲍娟, 张忠妍, 林东红, 精细石油化工, 2008, 25, 19.)

[15] (a) Kuroda, H.; Kubo, S.; Chino, N.; Kimura, T.; Sakakibara, S. Int. J. Pept. Protein Res. 1992, 40, 114.

(b) Ebbers, E. J.; Arianas, G. J.; Houbiers, J. P. M. Tetrahedron 1997, 53, 9417.

(c) Fang, B.-Y.; Feng, D.-Y. Chem. Res. Appl. 1997, 9, 40 (in Chinese).

(方百盈, 冯大炎, 化学研究与应用, 1997, 9, 40.)

(d) Liu, Y.; Guo, L.-F.; Wu, X.-Y.; Qiao, Q.-C. Chemistry 2005, 12, 935 (in Chinese).

(刘毅，郭丽芳，吴晓燕，焦庆才，化学通报, 2005, 12, 935.) 